\title{
A FLASH back to radiotherapy's past and then fast forward to the future
}

\begin{abstract}
Over the past decades, radiotherapy (RT) has received massive improvements that enabled radiation oncologists to escalate the dose to gross tumor volume and, at the same time, spare nearby organs. Nevertheless, local failures still pose a problem and the presence of dose-limiting normal tissue complications precludes further treatment intensification. In this context, ultra-high dose radiotherapy, also known as FLASH-RT, is emerging as a promising technology due to its differential effect on tumor vs. normal tissues. Although first described in 1967, preclinical studies on animal models have only recently confirmed its safety and effectiveness compared to conventional RT. In this short review, we outline the main preclinical data, discuss the case of the first patient that was treated with FLASH$\mathrm{RT}$ and contemplate on the hypotheses regarding its underlying mechanism.
\end{abstract}

Keywords: FLASH radiotherapy, ultra-high dose radiotherapy
Volume 10 Issue 6 - 2019

\author{
Efstathios Kamperis,' Chionia Kodona, ${ }^{2}$ \\ Vasileios Giannouzakos' \\ 'Department of Radiation Oncology, Papageorgiou General \\ Hospital, Greece \\ 2Department of Medical Physics, Papageorgiou General Hospital, \\ Greece
Correspondence: Efstathios Kamperis, Department of Radiation Oncology, Papageorgiou General Hospital, Agiou Pavlou 76, PO 564 29, Thessaloniki, Greece, Tel 231332 3417, Email ekamperi@gmail.com

Received: October 25, 2019 | Published: November 13, 2019

\section{Introduction}

Radiotherapy is an integral component of cancer management with more than half of patients receiving it at some point in their course of disease. During the past decades, the therapeutic ratio of radiotherapy has been steadily improving. ${ }^{1,2}$ First, by the empirical realization that fractionated irradiation allows for more normal tissue repair. And, second, by conforming the dose to the target as much

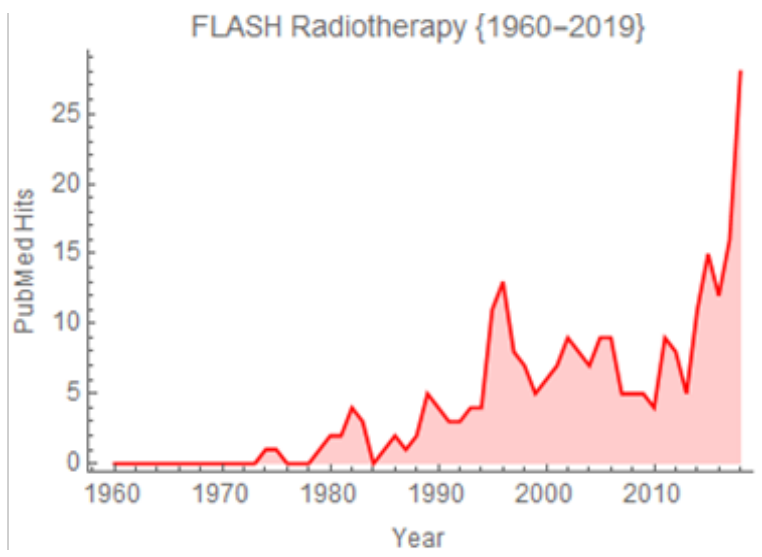

Figure I PubMed occurrences of "FLASH radiotherapy" terms.

FLASH-RT is the irradiation with ultrahigh dose rates, i.e. $>40 \mathrm{~Gy} /$ sec and even $>1000 \mathrm{~Gy} / \mathrm{sec}$. In comparison, a state of the art flatteningfilter free linear accelerator would irradiate at a rate of $\sim 0.2 \mathrm{~Gy} / \mathrm{sec}$ in an intensity modulated radiotherapy plan. Besides mean dose rate, though, other parameters are also important for invoking the "FLASH effect", such as the number of pulses (1-10) and the dose rate within the pulse (in the order of $105-106 \mathrm{~Gy} / \mathrm{sec}$ ). ${ }^{6}$

At the moment there is a debate whether FLASH irradiation represents the most significant discovery in recent radiotherapy history. Some researchers are skeptical regarding the efficacy of FLASH in terms of its technical complexity and our lack of understanding of its radiobiological underpinnings. ${ }^{7}$ For instance, before large scale as technically feasible, with $3 \mathrm{D}$ conformal radiotherapy and intensity modulated radiotherapy treatment variants. ${ }^{3,4} \mathrm{~A}$ third pillar has recently emerged, involving the delivery of irradiation with extremely high dose rates, known as FLASH-RT (Figure 1). The advantages of FLASH radiotherapy compared to conventional RT include increased organ sparing ( $\sim 30-35 \%$ of the prescribed dose), equivalent antitumor effect and "freeze" of target's physiological motion, resulting in a superior therapeutic index. ${ }^{5}$

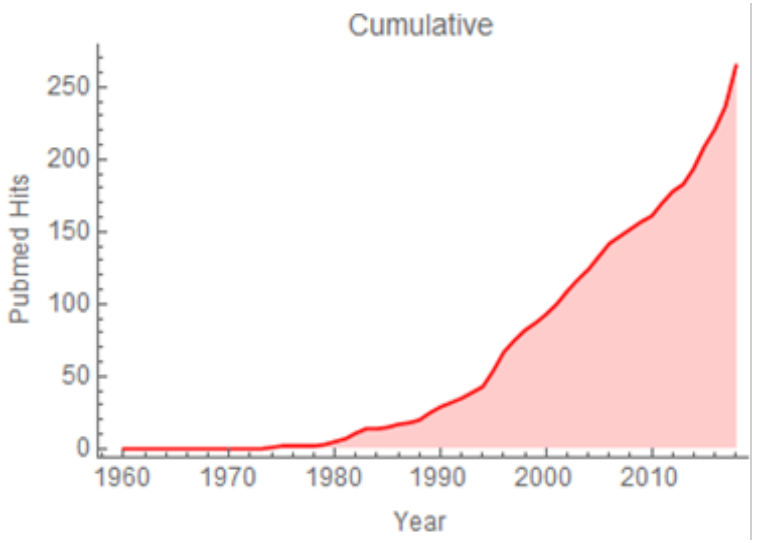

adoption of FLASH-RT is considered, certain safety issues need to be addressed, such as the implementation of dose monitoring and stopping system that can operate at a pulse level. ${ }^{6}$

\section{The first report}

The first report about the effects of high dose rate on the survival of mammalian cells is from Town in $1967 .{ }^{8}$ Town used a $15 \mathrm{MeV}$ Mullard linear accelerator at St. Bartholomew's Hospital to produce an electron beam in $1.3 \mu \mathrm{sec}$ pulses. The maximum dose that he used was $4500 \mathrm{rads}$ in one pulse of $1.3 \mu \mathrm{sec}$, corresponding to a dose rate of $\sim 3.5 \times 10^{9} \mathrm{rads} / \mathrm{sec}$ (or $3.5 \times 10^{7} \mathrm{~Gy} / \mathrm{sec}$ ). By irradiating a sub-clone of HeLa S3 cells with two different dose rates (single pulse vs. two 
pulses separated by $2.5 \mathrm{msec}$ ) he noted that after 900rads the survival curves started to separate. The cells that were irradiated with a single pulse showed reduced radiosensitivity. This phenomenon was "rediscovered" more than three decades later and during the past five years the research has been revived. ${ }^{6,9}$

The FLASH phenomenon has been observed across many research centers (Grenoble, Lausanne, Orsay and Stanford) ${ }^{9-12}$ with electron, ${ }^{9}$ photon $^{11}$ and proton beams, ${ }^{13,14}$ albeit most studies use low energy electrons. Conventional linear accelerators can be modified ${ }^{15}$ to deliver electron beams with dose rates $>200 \mathrm{~Gy} / \mathrm{sec}$, but the geometric properties of these beams are fit only for small-field experiments in animal models. The eRT6 "Oriatron" linear accelerator at the University Hospital of Lausanne can deliver FLASH-RT with an open electron field size of $20 \mathrm{~cm}$ diameter and $5.6 \mathrm{MeV}$ energy. ${ }^{6}$ In order to treat deeply seated tumors one needs very high energy electrons, FLASH-X-ray or even protons. The most promising technology for implementing FLASH-X-rays is the Pludirectional High-energy Agile Scanning Electronic Radiotherapy (PHASER).

\section{Mechanism of action}

The underlying mechanism implicates oxygen depletion when a high total dose is delivered at a very high dose rate. ${ }^{16}$ The reduction of oxygen cannot be compensated via diffusion and re-oxygenation, rendering the normal tissue hypoxic hence radioresistant. ${ }^{17}$ On the other hand, most tumors are already hypoxic, therefore the impact of oxygen depletion is presumably small. ${ }^{6}$ This is why FLASH should ideally be given at the beginning of the treatment, when the tumor is inherently hypoxic, as a "boost in advance" in the range of 20-25Gy, followed by conventional RT. ${ }^{6}$ Also, the total dose must be sufficiently large and the dose rate sufficiently high in order to consume local oxygen and elicit a FLASH effect. ${ }^{2}$

Although oxygen depletion is the most prevalent underlying mechanism, there might be the case that other factors also contribute to the manifestation of FLASH. Such examples could include differences in redox biology and immune-related effects (sparing of peripheral lymphocytes, massive release of neoantigens, sparing of intratumoral T cells, etc.). ${ }^{2,7,18}$

\section{The preclinical studies}

Preclinical studies include experiments on mice, cats, mini-pigs and zebrafish.,19 The sparing of organs via ultra-high irradiation has been demonstrated mainly with regard to brain, lung, skin and gastrointestinal tract. Simmons et al compared whole-brain radiation (WBI) in mice with conventional dose rates $(240 \mathrm{sec})$ and FLASH $(0.1-0.16 \mathrm{sec}) .{ }^{20}$ Interestingly, the following results were obtained at ten weeks post irradiation: 1. FLASH-RT preserved dendritic spines in hippocampal neurons. 2. It did not activate $\mathrm{CD}-68$ positive microglia and 3. Five out of 10 studied inflammation markers were significantly reduced in the experimental arm (and non-significant reductions were seen in another 3).

The hypothesis that oxygen depletion is the basis of the sparing effect is corroborated by certain preclinical findings. Increasing the oxygen tension in the brain, via carbogen breathing, cancelled the neuroprotective effect of FLASH in WBI of mice. ${ }^{12}$ Similar results have previously been obtained by Montay-Gruel et al. ${ }^{21}$ where neurocognitive toxicity after WBI was reduced with increased dose rate. Concretely, spatial memory was preserved after WBI with mean dose rates above $100 \mathrm{~Gy} / \mathrm{sec}$, whereas $10 \mathrm{~Gy} \mathrm{WBI}$ at a conventional RT dose rate $(0.1 \mathrm{~Gy} / \mathrm{sec})$ totally impaired it.

Favaudon et al. ${ }^{9}$ investigated lung fibrogenesis in C57BL/6J mice irradiated at different dose rates $(\geq 40 \mathrm{~Gy} / \mathrm{sec}$ or $\leq 0.03 \mathrm{~Gy} / \mathrm{sec})$ in a single fraction. Conventional RT (15Gy) triggered lung fibrosis associated with activation of the Transforming Growth Factor- $\beta$ (TGF- $\beta$ ) cascade. On the contrary, no complications developed for more than 36 weeks following FLASH-RT below 20Gy. Also, FLASH-RT was equally efficient as conventional RT, with regard to tumor growth inhibition.

The advantages of FLASH-RT have also been reproduced in the skin of mini-pigs. ${ }^{2}$ Single fractions of doses 22-34Gy were prescribed to an area with $2.6 \mathrm{~cm}$ diameter. At 9 months there were no signs of late skin necrosis and $25 \mathrm{~Gy}$ of conventional RT yielded similar results to $34 \mathrm{~Gy}$ delivered with FLASH-RT, suggesting that a factor $\sim 1.36$ of sparing was attainable. Even with a dose of $31 \mathrm{~Gy}$ at a treated area of $8 \times 8 \mathrm{~cm}^{2}$, only transient ulceration was observed that was healed spontaneously later on.

Schueler et al. ${ }^{22}$ irradiated the gastrointestinal tract of C57BL/6 mice with doses ranging between $10-18 \mathrm{~Gy}$ with $20 \mathrm{MeV}$ electrons. Following irradiation, mice were monitored twice daily for morbidity and daily for weight changes. Those irradiated with FLASH-RT had lower weight loss compared to the mice receiving conventional RT. As far as survival is concerned, all mice in the conventional RT groups had a $100 \%$ mortality in the range of $15.5-18 \mathrm{~Gy}$, while mice in the FLASH-RT group had a $100 \%$ survival in the same range.

FLASH's antitumor effect has been demonstrated in lung, breast, head and neck and glioblastoma tumor models. ${ }^{9,13,21}$ Cats with spontaneous squamous cell cancer of nasal planum were treated with doses 25-41Gy and volumes in the range 6 to $25 \mathrm{ml} .^{2,19}$ The maximum tolerated dose was not reached and control rate was $84 \%$ at 1 year.

\section{The first human patient}

Given the consistent normal tissue sparing across many different animal models, the magnitude of the differential effect and the preservation of the antitumor effect, the transition to clinical trials in humans is justified6. The first patient to undergo FLASH irradiation was a 75years old man with T-cell lymphoma at Lausanne University hospital. He had received 110 irradiations to various sites on top of the administered systemic therapies. Unfortunately, he developed a new $3.5 \mathrm{~cm}$ painful lesion in his forearm and underwent FLASH radiotherapy with a $5.6 \mathrm{MeV}$ electron beam at a total dose of $15 \mathrm{~Gy}$ in one fraction of $90 \mathrm{msec}$, corresponding to a mean dose rate of $\sim 167 \mathrm{~Gy} /$ sec. During the post-RT period he developed asymptomatic transient edema (Grade 1 per NCI-CTCAE v. 5.0) that peaked the 15th day. An optical coherence tomography of the irradiated skin showed neither disruption of the dermis - epidermis junction nor any other toxicity besides an edema of the epidermis. The patient was followed-up and at 5 months a complete response of the lesion was documented and no late side effects whatsoever.

\section{Conclusion}

The delivery of ultra-high dose radiotherapy enhances the differential effect between antitumor effect and normal tissue complications. The underlying mechanism implies the generation of transient protective hypoxia through oxygen depletion. Mean dose rates larger than $100 \mathrm{~Gy} / \mathrm{sec}$ may be needed to fully exploit the 
FLASH effect. There is a need for further preclinical trials to study the antitumor effect and conclude on the optimal physical parameters (number of pulses, dose rate within the pulses, mean dose rate, beam type, field size, etc.).

\section{Acknowledgments}

None.

\section{Conflicts of interest}

The authors declare there are no conflicts of interest.

\section{References}

1. Bernier J, Hall EJ, Giaccia A. Radiation oncology: a century of achievements. Nat Rev Cancer. 2004;4(9):737-747.

2. Vozenin MC, Hendry JH, Limoli CL. Biological benefits of ultra-high dose rate FLASH radiotherapy: sleeping beauty awoken. Clin Oncol. 2019;31(7):407-415.

3. Dawson LA, Jaffray DA. Advances in image-guided radiation therapy. $J$ Clin Oncol. 2007;25(8):938-946.

4. Palma DA, Verbakel WFAR, Otto K, et al. New developments in arc radiation therapy: a review. Cancer Treat Rev. 2010;36(5):393-399.

5. Maxim PG, Tantawi SG, Loo BW Jr. PHASER: A platform for clinical translation of FLASH cancer radiotherapy. Radiother Oncol. 2019;139:28-33.

6. Bourhis J, Montay-Gruel P, Gonçalves Jorge P, et al. Clinical translation of FLASH radiotherapy: Why and how? Radiother Oncol. 2019;139:1117.

7. Maxim PG, Keall P, Cai J. FLASH radiotherapy: Newsflash or flash in the pan? Med Phys. 2019;46(10):4287-4290.

8. Town CD. Radiobiology. Effect of high dose rates on survival of mammalian cells. Nature. 1967;215(5103):847-848.

9. Favaudon V, Caplier L, Monceau V, et al. Ultrahigh dose-rate FLASH irradiation increases the differential response between normal and tumor tissue in mice. Sci Transl Med. 2014;6(245):245ra93.

10. Schüler E, Trovati S, King G, et al. Experimental platform for ultra-high dose rate FLASH irradiation of small animals using a clinical linear accelerator. Int J Radiat Oncol Biol Phys. 2017;97(1):195-203.
11. Montay-Gruel P, Bouchet A, Jaccard M, et al. X-rays can trigger the FLASH effect: Ultra-high dose-rate synchrotron light source prevents normal brain injury after whole brain irradiation in mice. Radiother Oncol. 2018;129(3):582-588.

12. Montay-Gruel P, Acharya MM, Petersson K, et al. Long-term neurocognitive benefits of FLASH radiotherapy driven by reduced reactive oxygen species. Proc Natl Acad Sci USA. 2019;116(22):1094310951.

13. Zlobinskaya O, Siebenwirth $\mathrm{C}$, Greubel C, et al. The effects of ultra-high dose rate proton irradiation on growth delay in the treatment of human tumor xenografts in nude mice. Radiat Res. 2014;181(2):177-183.

14. Buonanno M, Grilj V, Brenner DJ. Biological effects in normal cells exposed to FLASH dose rate protons. Radiother Oncol. 2019;139:51-55.

15. Lempart M, Blad B, Adrian G, et al. Modifying a clinical linear accelerator for delivery of ultra-high dose rate irradiation. Radiother Oncol. 2019;139:40-45.

16. Hornsey S, Bewley DK. Hypoxia in mouse intestine induced by electron irradiation at high dose-rates. Int J Radiat Biol Relat Stud Phys Chem Med. 1971;19(5):479-483.

17. Durante M, Bräuer-Krisch E, Hill M. Faster and safer? FLASH ultrahigh dose rate in radiotherapy. Br J Radiol. 2018;91(1082):20170628.

18. Spitz DR, Buettner GR, Petronek MS, et al. An integrated physicochemical approach for explaining the differential impact of FLASH versus conventional dose rate irradiation on cancer and normal tissue responses. Radiother Oncol. 2019;139:23-27.

19. Vozenin MC, De Fornel P, Petersson K, et al. The Advantage of FLASH Radiotherapy Confirmed in Mini-pig and Cat-cancer Patients. Clin Cancer Res. 2019;25(1):35-42.

20. Simmons DA, Lartey FM, Schüler E, et al. Reduced cognitive deficits after FLASH irradiation of whole mouse brain are associated with less hippocampal dendritic spine loss and neuroinflammation. Radiother Oncol. 2019;139:4-10.

21. Montay-Gruel P, Petersson $\mathrm{K}$, Jaccard $\mathrm{M}$, et al. Irradiation in a flash: Unique sparing of memory in mice after whole brain irradiation with dose rates above 100Gy/s. Radiother Oncol. 2017;124(3):365-369.

22. Schueler E, Trovati S, King G, et al. TU-H-CAMPUS-TeP2-02: FLASH irradiation improves the therapeutic index following GI tract irradiation. Medical Physics. 2016;43(6):3783-3783. 